\title{
Microscopic structure and mathematical background of the multiconfigurational dynamical symmetry
}

\author{
J. Cseh $\odot$ \\ Institute for Nuclear Research, Debrecen, $P O B$ 51, Hungary-4001
}

(Received 2 September 2020; revised 2 March 2021; accepted 26 May 2021; published 21 June 2021)

\begin{abstract}
Background: The multiconfigurational dynamical symmetry (MUSY) is the common intersection of the shell, collective, and cluster models for the multi-major-shell problem. It is able to describe the spectra of different configurations in different energy windows in a unified way. It is based on some heuristic arguments, related to the connection of the wave functions, and energy eigenvalues. The detailed mathematical background has been worked out so far only for the simplest case of the two binary cluster configurations.

Purpose: I intend to construct the exact algebraic framework for the general case of the MUSY, i.e., for any number of configurations and any number of clusters or nucleons. As an illustrative example, the spectrum of different configurations of the ${ }^{44} \mathrm{Ti}$ nucleus is described by a simple Hamiltonian.

Methods: Classification schemes defined by different algebra chains need to be combined; in particular, those of the major shell scheme and of the particle index scheme.

Results: A class of Hamiltonians, which is invariant under the transformations from one configuration to the other, is determined. In case of the ${ }^{44} \mathrm{Ti}$ low-lying shell-model spectrum, as well as ${ }^{40} \mathrm{Ca}+{ }^{4} \mathrm{He}$ and ${ }^{28} \mathrm{Si}+{ }^{16} \mathrm{O}$ cluster states are obtained in a unified way.

Conclusions: The MUSY is based on two pillars: (i) a unified multiplet structure for shell, collective, and cluster model states and (ii) a Hamiltonian which is invariant with respect to the transformations from one configuration to the other.
\end{abstract}

DOI: 10.1103/PhysRevC.103.064322

\section{INTRODUCTION}

The multiconfigurational dynamical symmetry (MUSY) $[1,2]$ is a unifying symmetry that connects the fundamental structure models of atomic nuclei: the shell, collective, and cluster models [3]. It is a composite symmetry in the sense that each configuration has a usual [U(3)] dynamical symmetry, and in addition a further symmetry transforms these configurations into each other. Apart from its interesting theoretical aspects, the MUSY also has some practical usage. The latter feature is a consequence of the fact that it connects the wave functions, as well as the complete energy spectra of different configurations. Therefore, it has some predictive power.

E.g., one can find the shape isomers of light nuclei, based on a symmetry-self-consistency argument [4] (which is an alternative method to the well-known energy-minimum calculation). Then the MUSY defines the allowed binary cluster configurations of the shape isomers; i.e., it provides us with those reaction channels, which can populate these extremely deformed states, or in which they can decay. In some cases

Published by the American Physical Society under the terms of the Creative Commons Attribution 4.0 International license. Further distribution of this work must maintain attribution to the author(s) and the published article's title, journal citation, and DOI. this kind of predictions has been approved by experimental observations [5,6].

The unified description of different spectra can also lead to highly nontrivial results. In [7], e.g., the high-lying ${ }^{12} \mathrm{C}+{ }^{16} \mathrm{O}$ cluster spectrum was obtained as a parameter-free prediction from the quartet model description of the well-established low-lying bands of the ${ }^{28} \mathrm{Si}$ nucleus. The model spectrum turned out to be in good agreement with the observed energy spectrum of the fine-resolution ${ }^{12} \mathrm{C}+{ }^{16} \mathrm{O}$ molecular resonances. This calculation answered the long-standing question on these heavy-ion resonances: they form the spectrum of the second (superdeformed) minimum of the energy surface. The same (parameter-free) dynamical symmetry gives the distribution of the high-lying $0^{+}$states of ${ }^{28} \mathrm{Si}$, again in good agreement with the experimental results of the ${ }^{24} \mathrm{Mg}+{ }^{4} \mathrm{He}$ reaction study [8].

The MUSY was first introduced as a connecting symmetry of different cluster configurations [1]. Therefore, it was also called multichannel dynamical symmetry, referring to the reaction channels which define the cluster configurations. The reasoning for this possible composite symmetry was based on the connection of the wave functions, and as a consequence on the relations of the energy eigenvalues. The explicit transformations between the different configurations were studied so far only for the simple case of the two binary cluster configurations in [2].

Later on it was realized that the shell model configuration [9] is also related to the cluster configurations in the same way, 
i.e., how the different clusterizations are connected [7]. In fact quartet configurations were considered, which are special shell configurations [9]. (Strictly speaking a quartet state is a special shell state when one speaks about shell-like quarteting. Note, however, that other interpretations are also present in the literature.)

Here I present the full mathematical background of the multiconfigurational dynamical symmetry. First I consider the wave-function and eigenvalue connections, then the algebraic structure of this composite symmetry is shown in detail. Both argumentations are presented for the general problem: without having any restrictions on the configurations, their number as well as their type can be arbitrary.

In particular: in the next section the shell scheme is discussed, which provides us also with a scenario for the construction of the full basis of the Hilbert space. Then I consider how the total space should be truncated in order to obtain smaller model spaces, relevant for the quadrupole collectivity, or clusterization. I find that all these truncations can be done according to the irreducible representations (irreps) of a single group chain. It defines the algebraic structure of the MUSY, and with a corresponding Hamiltonian the connection between the different configurations is established. But it does not illuminate the connecting symmetry of the configurations yet. In Sec. V, I discuss this problem and present the coupling scheme, which contains these transformations explicitly. In Sec. VI the MUSY is applied for the unified description of the quartet configuration, as well as of the ${ }^{40} \mathrm{Ca}+{ }^{4} \mathrm{He}$ and ${ }^{28} \mathrm{Si}+{ }^{16} \mathrm{O}$ cluster configurations in the ${ }^{44} \mathrm{Ti}$ nucleus.

\section{SHELL SCHEME}

Here I consider a coupling scheme of the many nucleon system, which is governed by the shell structure in the sense that the total wave function is built up from those of the major shells. In doing so I show an actual scenario for the construction of the shell model space, too.

I apply an $L-S$ coupling scheme. The operators are built from the oscillator quantum creation $(\eta)$ and annihilation $(\xi)$ operators. They have three components: $j=1,2,3$ (e.g., the three Cartesian coordinates) and carry the particle index $s=$ $1, \ldots, n: \eta_{j}^{s}, \xi_{j}^{s}$. Their number conserving bilinear products are $C_{j k}^{s t}=\eta_{j}^{s}$, $\xi_{k}^{t}$. In the shell scheme one sums up with respect to the particle index [10]:

$$
C_{j k}=\sum_{s=1}^{n} C_{j k}^{s s} .
$$

The commutation relations are

$$
\left[C_{j k}, C_{j^{\prime} k^{\prime}}\right]=\delta_{k j^{\prime}} C_{j k^{\prime}}-\delta_{j k^{\prime}} C_{j^{\prime} k} .
$$

The linear combinations of these operators form a Lie algebra, and they generate the U(3) group.

The Hamiltonian of the harmonic oscillator $(\mathrm{HO})$ is

$$
H_{\mathrm{HO}}=\sum_{k=1}^{3} C_{k k} .
$$

Each $C_{j k}$ commutes with it, thus $\mathrm{U}(3)$ is the symmetry group of the harmonic oscillator.
The angular momentum is a good quantum number of the nuclear states; therefore, the physically relevant group chain contains $\mathrm{SO}(3)$ :

$$
\begin{array}{cl}
\mathrm{U}(3) & \supset \mathrm{SU}(3) \supset \mathrm{SO}(3) \supset \mathrm{SO}(2) \\
{\left[n_{1}, n_{2}, n_{3}\right],(\lambda, \mu), K, \quad L,} & M .
\end{array}
$$

Here I indicated the representation labels, too.

The spin-isospin degrees of freedom are described by Wigner's $U^{S T}(4)$ group, and its spin and isospin subgroups: $\mathrm{U}^{S T}(4) \supset \mathrm{SU}^{S}(2) \otimes \mathrm{SU}^{T}$ (2) [11]. (I leave the details of the spin-isospin formalism for Sec. V.)

\section{A. Single major shell}

Let us consider $m$ particles in a single harmonic oscillator major shell which has $N$ orbitals. Then $\mathrm{U}(\mathrm{N})$ and the permutation group $\mathrm{S}(\mathrm{m})$ have representations sharing the same Young pattern. The total antisymmetry of the many-body wave function requires that $\mathrm{U}(\mathrm{N})$ and $\mathrm{U}^{S T}(4)$ have associate representations, i.e., their Young patterns are obtained from each other by reflection: interchanging the rows and columns [12]. The group symmetry of the single shell problem is

$$
\begin{array}{r}
\left\{\mathrm{U}^{S T}(4) \supset \mathrm{SU}^{S}(2) \otimes \mathrm{SU}^{T}(2)\right\} \otimes \\
\{\mathrm{U}(\mathrm{N}) \supset \mathrm{U}(3) \supset \mathrm{SU}(3) \supset \mathrm{SO}(3)\} .
\end{array}
$$

The relevant $\mathrm{U}(3)$ [and $\mathrm{SU}(3)$ ] representations are determined by those of the $\mathrm{U}(\mathrm{N})$ group [13].

More general Hamiltonians (than that of the HO) still having U(3) dynamical symmetry and analytical solvability can also be obtained. They are expressed in terms of the $C_{i j}$ operators. The $C_{i j}$ 's can be recast into spherical tensors by taking proper linear combinations. The angular momentum $L$ is a vector operator (of three components), and the mass quadrupole operator $Q^{a}$ is a second rank tensor (of five components). (I apply here the $a$ superscript in order to distinguish this single shell operator from the physical quadrupole momentum, which contains major shell excitations, too, as given later on.)

One can easily construct a dynamically symmetric Hamiltonian,

$$
H=C_{\mathrm{U} 3}^{(1)}+\alpha C_{\mathrm{SU} 3}^{(2)}+\delta C_{\mathrm{SO} 3}^{(2)},
$$

where $C^{(i)}$ stands for the Casimir invariant of degree $i$ of the algebra indicated as a subscript:

$$
C_{\mathrm{U} 3}^{(1)}=n, \quad C_{\mathrm{SU} 3}^{(2)}=\frac{3}{4} L L+\frac{1}{4} Q^{a} Q^{a}, \quad C_{\mathrm{SO} 3}^{(2)}=L L,
$$

where $n=n_{1}+n_{2}+n_{3}$ is the number of oscillator quanta. This operator is diagonal in the U(3) basis. The original energy operator of the Elliott model [10] has also this form (with a fixed ratio of $\alpha$ and $\delta$ to give the quadrupole force).

\section{B. Many major shells}

When major shell excitations take place then the scenario of the single shell problem is repeated several times, and the 
group structure is

$$
\begin{array}{r}
\left\{\mathrm{U}_{1}^{S T}(4) \otimes \mathrm{U}_{2}^{S T}(4) \otimes \ldots \mathrm{U}_{k}^{S T}(4)\right\} \otimes \\
\left\{\mathrm{U}\left(\mathrm{N}_{1}\right) \otimes \mathrm{U}\left(\mathrm{N}_{2}\right) \otimes \ldots \mathrm{U}\left(\mathrm{N}_{\mathrm{k}}\right)\right\},
\end{array}
$$

where $k$ stands for the number of major shells relevant in the problem.

In order to have a fully antisymmetric many-body wave function one determines the $\mathrm{U}(3)$ representations as follows. The construction is carried out for each sector of the $\mathrm{U}^{S T}(4)$ spin-isospin group, separately.

(i) In the first step one determines those $\mathrm{U}^{S T}$ (4) irreps of the different major shells, which provide us with the relevant spin-isospin symmetry of the whole system as their outer product.

(ii) These $\mathrm{U}^{S T}(4)$ irreps determine the relevant $\mathrm{U}(\mathrm{N})$ irreps in each major shell (by their associate Young pattern).

(iii) Those U(3) irreps are taken into account in the construction procedure, which belong to the relevant $\mathrm{U}(\mathrm{N})$ [and $\left.\mathrm{U}^{S T}(4)\right]$ representations.

In addition one has to remove the spurious excitations of the center of mass, which can be done, e.g., according to the procedure of [14].

The shell model space constructed in this way provides us with a complete basis of the Hilbert space of the many nucleon system. Therefore, the wave function of any state can be expanded in this basis.

In the next section, I consider those sectors of the full space which are especially important from the viewpoint of the quadrupole collectivity and clusterization.

\section{COLLECTIVE AND CLUSTER SECTIONS}

The quadrupole deformation and the collective rotation of the nuclei were understood from the viewpoint of the spherical shell model for the first time in the Elliott model [10]. In its original version it is a single shell model. The quadrupole shape of the intrinsic state is given by the $(\lambda, \mu) \mathrm{SU}(3)$ labels. The $(0,0)$ representation corresponds to the spherical shape, $(\lambda, 0)$ indicates a prolate shape with cylindrical symmetry, $(0, \mu)$ is oblate, while $\lambda \neq 0, \mu \neq 0$ stands for a triaxial nucleus. The $\beta$ and $\gamma$ deformation parameters are determined by the $(\lambda, \mu)$ representation labels [15]:

$$
\beta^{2}=\frac{16 \pi}{5 N_{0}^{2}}\left(\lambda^{2}+\mu^{2}+\lambda \mu\right), \gamma=\arctan \left(\frac{\sqrt{3} \mu}{2 \lambda+\mu}\right) .
$$

Here $N_{0}$ is the number of oscillator quanta, including the zero point contribution $N_{0}=n+(A-1) \frac{3}{2}, n$ is the sum of the $\mathrm{U}(3)$ quantum numbers $n=n_{1}+n_{2}+n_{3}$, and $A$ is the mass number of the nucleus.

Due to the close relation between the SU(3) symmetry and the quantum rotor [16] the quadrupole deformation and rotation can be described already in a single shell model. This treatment, however, requires an effective charge for the reproduction of the electric quadrupole transition. The more fundamental description, without effective charge, incorporates major shell excitations.

\section{A. Symplectic shell model}

The symplectic shell model [17] proved to be very useful for this purpose. The $\mathrm{Sp}(6, \mathrm{R})$ group is generated by the position vectors and their canonically conjugate momenta of the nucleons. [I note here that the language of the literature is not univocal; some authors denote this group by $\operatorname{Sp}(3, \mathrm{R})]$. An alternative set of its generator is expressed in terms of harmonic oscillator operators, containing the nine generators of the U(3) group (which preserve the number of oscillator quanta), and in addition six creation $B_{j, k}^{\dagger}=\sum_{s=1}^{n} \eta_{j}^{s}, \eta_{k}^{s}$, and six annihilation $B_{j, k}=\sum_{s=1}^{n} \xi_{j}^{s}, \xi_{k}^{s}$ operators, which ladder by 2 or -2 quanta. The creation operators are $[2,0,0] \mathrm{U}(3)$ tensors; therefore, their products also carry $\mathrm{U}(3)$ labels: $\left[n_{1}^{e}, n_{2}^{e}, n_{3}^{e}\right]$. The relevant group structure of this model is

$$
\begin{aligned}
& \mathrm{Sp}(6, \mathrm{R}) \quad \supset \mathrm{U}(3) \quad \supset \mathrm{SU}(3) \supset \mathrm{SO}(3) \\
& \left|\left[n_{1}^{s}, n_{2}^{s}, n_{3}^{s}\right],\left[n_{1}^{e}, n_{2}^{e}, n_{3}^{e}\right], \rho,\left[n_{1}, n_{2}, n_{3}\right],(\lambda, \mu), K, L\right\rangle \text {. }
\end{aligned}
$$

Here $\left[n_{1}^{s}, n_{2}^{s}, n_{3}^{s}\right]$ denotes the symplectic bandhead, which is a $\mathrm{U}(3)$ irrep, being a lowest-weight $\mathrm{Sp}(6, \mathrm{R})$ state, while $\rho$ distinguishes multiple occurrences of $\left[n_{1}, n_{2}, n_{3}\right]$ in the product $\left[n_{1}^{e}, n_{2}^{e}, n_{3}^{e}\right] \otimes\left[n_{1}^{s}, n_{2}^{s}, n_{3}^{s}\right]$.

In collective terms the symplectic model includes monopole and quadrupole vibrations as well as vorticity degrees of freedom for the description of the rotational dynamics in a continuous range from the irrotational flow to the rigid rotor.

\section{B. Contracted symplectic model}

In the large $n$ limit the symplectic shell model reduces to a collective model with a simpler structure. The dynamical group of the model simplifies to $\mathrm{U}_{b}(6) \otimes \mathrm{U}_{s}(3)$, i.e., to a compact group, as opposed to the noncompact $\operatorname{Sp}(6, \mathrm{R})$ of the shell model. Technically it is achieved by replacing the raising and lowering operators by boson creation and annihilation operators: $b_{j k}^{\dagger}=(1 / \epsilon) B_{j k}^{\dagger}, b_{j k}=(1 / \epsilon) B_{j k}$, where $\epsilon$ denotes $\left[\frac{4}{3} n_{s}\right]^{\frac{1}{2}} ; n_{s}=n_{1}^{s}+n_{2}^{s}+n_{3}^{s}$. This model is called the U(3) boson model [18], or contracted symplectic model [19]. Mathematical justification for the simplifying assumptions is provided through the application of the group deformation mechanism. This model is more easily applicable, e.g., it has an orthonormal set of basis states defined by the group chain:

$$
\mathrm{U}_{s}(3) \otimes \mathrm{U}_{b}(6) \supset \mathrm{U}_{s}(3) \otimes \mathrm{U}_{b}(3) \supset \mathrm{U}(3) \supset \mathrm{SU}(3) \supset \mathrm{SO}(3) .
$$

The united U(3) group is generated by the sum of the operators corresponding to the subgroups $\mathrm{U}_{s}(3) \otimes \mathrm{U}_{b}(3)$ :

$$
n=n_{s}+2 n_{b}, Q=Q_{s}+Q_{b}, L=L_{s}+L_{b} .
$$

The $\mathrm{U}_{s}(3)$ is Elliott's shell model symmetry of the $0 \hbar \omega$ shell, and $\mathrm{U}_{b}(6)$ is the group of the six dimensional oscillator, generated by the bilinear products of the $(l=0$ and 2$)$ boson creation and annihilation operators. It is realized in a similar way as the $\mathrm{U}(6)$ group of the interacting boson model [20]; nevertheless physically it is different, because in the case of the contracted symplectic model the bosons are associated to intershell excitations, not to intrashell ones. 


\section{Semimicroscopic algebraic cluster model}

This model combines a microscopic model space with fully algebraic description, i.e., not only the basis states, but also the operators carry group symmetry [21]. The internal structure of the clusters is described here by the Elliott model [10]; therefore, this part of the wave function has a $\mathrm{U}_{C}^{S T}(4) \otimes \mathrm{U}_{C}(3)$ symmetry. The relative motion of the clusters is accounted for by the modified $\left[\mathrm{U}_{R}(4)\right]$ vibron model [22].

The coupling between the relative motion and internal cluster degrees of freedom for a binary cluster system results in a group structure:

$$
\mathrm{U}_{C_{1}}^{S T}(4) \otimes \mathrm{U}_{C_{1}}(3) \otimes \mathrm{U}_{C_{2}}^{S T}(4) \otimes \mathrm{U}_{C_{2}}(3) \otimes \mathrm{U}_{R}(4) .
$$

The spin and isospin degrees of freedom are essential from the viewpoint of the construction of the model space. However, if one is interested only in a single supermultiplet $\left[\mathrm{U}_{C}^{S T}(4)\right]$ symmetry, which is typical in cluster problems, then the relevant group structure simplifies to that of the space part. It is characterized by the group chain:

$$
\begin{array}{r}
\mathrm{U}_{C_{1}}(3) \otimes \mathrm{U}_{C_{2}}(3) \otimes \mathrm{U}_{R}(4) \supset \mathrm{U}_{C}(3) \otimes \mathrm{U}_{R}(3) \supset \\
\mathrm{U}(3) \supset \mathrm{SU}(3) \supset \mathrm{SO}(3),
\end{array}
$$

where $U_{C}(3)$ stands for the coupled space symmetry of the two clusters.

The model space is free from the Pauli-forbidden states and the spurious excitations of the center of mass; it is identical with those of the fully microscopic cluster models [when they apply the $\mathrm{U}^{S T}(4) \otimes \mathrm{U}(3)$ basis].

\section{MUSY: THE WAVE-FUNCTION CONNECTION}

As it is seen above, for the many major shell problem both the (symplectic) shell model and the (contracted symplectic) collective model, as well as the (microscopic or semimicroscopic algebraic) cluster model, have a set of basis states characterized by the irreps of the

$$
\begin{aligned}
& \mathrm{U}_{x}(3) \otimes \mathrm{U}_{y}(3) \quad \supset \mathrm{U}(3) \quad \supset \mathrm{SU}(3) \supset \mathrm{SO}(3) \\
& \left|\left[n_{1}^{x}, n_{2}^{x}, n_{3}^{x}\right],\left[n_{1}^{y}, n_{2}^{y}, n_{3}^{y}\right], \rho,\left[n_{1}, n_{2}, n_{3}\right], \quad(\lambda, \mu), K, L\right\rangle
\end{aligned}
$$

group chain. For the shell and collective models $x$ stands for the bandhead (i.e., the lowest-lying major shell configuration of the symplectic cone); for the cluster model it refers to the internal cluster structure. $y$ indicates in each case the major shell excitations; in the shell and collective model cases it takes place in steps of $2 \hbar \omega$, connecting oscillator shells of the same parity, while in the cluster case it is in steps of $1 \hbar \omega$, incorporating all the major shells. For the cluster model it has only completely symmetric (single-row Young tableaus) irreps: $[n, 0,0]$, while in the case of the shell and collective models it can be more general. As a consequence the model spaces of the three models have a considerable overlap, but they are not identical.

Let us consider a simple dynamically symmetric Hamiltonian

$$
H=C_{\mathrm{U} 3}^{(1)}+\alpha C_{\mathrm{SU} 3}^{(2)}+\beta C_{\mathrm{SU} 3}^{(3)}+\delta C_{\mathrm{SO} 3}^{(2)},
$$

in which the contribution of the shell excitation $\left[\mathrm{U}_{y}(3)\right]$ and the internal structure $\left[\mathrm{U}_{x}(3)\right]$ appears only in the coupled
[U(3)] form. The physical content of its members are as follows. $C_{\mathrm{U} 3}^{(1)}$ is the harmonic oscillator Hamiltonian [see Eq. (3)]. $C_{\mathrm{SU} 3}^{(2)}$ and $C_{\mathrm{SO} 3}^{(2)}$ involve the quadrupole and the angular momentum interactions, respectively [see Eq. (7)]. $C_{\mathrm{SU} 3}^{(3)}$ distinguishes between the prolate and oblate shape [7]. This operator proved to be successful in reproducing experimental spectra [7].

The analysis of the eigenvalue equation of this kind of Hamiltonian together with the relation of the wave functions explains the multiconfigurational dynamical symmetry.

As mentioned above, any cluster wave function can be expanded in terms of the shell model basis. This expansion simplifies considerably when the U(3) basis is applied. It was shown by Wildermuth and Kanellopoulos [23] that the Hamiltonians of the two models can be rewritten into each other exactly for harmonic oscillator interactions. (In fact the connection is valid also for the more general interactions, e.g., for those which have the present $U(3)$ dynamical symmetry with quadratic terms [24]). Thus any cluster state can be expanded in the shell basis of the same number of oscillator quanta. Furthermore, basis states of different U(3) representations are orthogonal to each other. Therefore, if a shell model state of a specific U(3) symmetry has a single multiplicity, then the cluster state (any cluster state) is identical with it (having only a single term in the expansion). Consequently the energy eigenvalue is the same in the shell (or quartet) and (any) cluster descriptions. This constraint defines a unique relation between the complete energy spectra.

That was the basic consideration for the introduction of the MUSY originally in order to connect different cluster configurations [1]. Later on it was realized that the shell configurations or their simplified versions, the quartet configurations, are related to the clusterization by the same symmetry [7,9]. Furthermore, due to the fact that the $U(3)$ symmetry determines the quadrupole shape, this dynamical symmetry turned out to be the common intersection of the shell, collective, and cluster models for the many major shell problem [3].

In relation to the Hamiltonian (16) it is worth mentioning that it shows the interesting phenomenon of the dual breaking of symmetries (similarly to many other dynamical symmetries of the algebraic models [25]). In particular, it can be written as a sum of the intrinsic $H_{\text {intr }}=C_{\mathrm{U} 3}^{(1)}+\alpha C_{\mathrm{SU} 3}^{(2)}$ and collective $H_{\text {coll }}=\delta C_{\mathrm{SO} 3}^{(2)}$ Hamiltonians: $H=H_{\text {intr }}+H_{\text {coll }}$. The first part defines the shape of the nucleus, and the energy of the bandheads, while the second one gives the rotational bands. The $\mathrm{U}(3)$ and $\mathrm{SU}(3)$ symmetries are dynamically broken by the collective part, while the $\mathrm{SO}(3)$ symmetry of the eigenvalue equation of the intrinsic part may be spontaneously broken (resulting in deformed nuclear shape) [26].

In the next section I show explicitly what is the connecting symmetry of the different configurations, i.e., I consider the transformations in the particle index space.

\section{MUSY: TRANSFORMATIONS IN THE PARTICLE SPACE}

\section{A. Particle scheme}

I consider here another coupling scheme, which carries the particle indices in the whole formalism. Its relevant features are recalled here, based on [27]. 
The $(3 n)^{2}$ number conserving bilinear products of the creation and annihilation operators $C_{j k}^{s t}=\eta_{j}^{s}$, $\xi_{k}^{t}$ satisfy the commutation relations:

$$
\begin{aligned}
{\left[C_{j k}^{s t}, C_{j^{\prime} k^{\prime}}^{s^{\prime} \prime^{\prime}}\right] } & =\delta^{t s^{\prime}} \delta_{k j^{\prime}} C_{j k^{\prime}}^{s t^{\prime}}-\delta^{s t t^{\prime}} \delta_{j k^{\prime}} C_{j^{\prime} k}^{s^{\prime} t}, \\
\left(C_{j k}^{s t}\right)^{\dagger} & =\left(C_{k j}^{t s}\right) .
\end{aligned}
$$

The linear combinations of these operators form a Lie algebra, and they generate the $\mathrm{U}(3 \mathrm{n})$ group. Each operator commutes with the HO Hamiltonian, thus $\mathrm{U}(3 \mathrm{n})$ is the symmetry group of the problem of $n$ nucleons in the HO potential.

From the mathematical viewpoint the summation with respect to the particle index of Eq. (1) is a contraction of the $\mathrm{U}(3 \mathrm{n})$ group. But it is not the only possible way to contract this group; it can be contracted also with respect to the coordinates (for the easier comparison I repeat here some equations):

$$
C_{j k}=\sum_{s=1}^{n} C_{j k}^{s s}, \quad C^{s t}=\sum_{j=1}^{3} C_{j j}^{s t} .
$$

The contracted operators satisfy the commutation relations:

$$
\begin{aligned}
{\left[C_{j k}, C_{j^{\prime} k^{\prime}}\right] } & =\delta_{k j^{\prime}} C_{j k^{\prime}}-\delta_{j k^{\prime}} C_{j^{\prime} k}, \\
{\left[C^{s t}, C^{s^{\prime} t^{\prime}}\right] } & =\delta^{t s^{\prime}} C^{s t^{\prime}}-\delta^{s t^{\prime}} C^{s^{\prime} t}, \\
{\left[C_{j k}, C^{s t}\right] } & =0 .
\end{aligned}
$$

Therefore, they generate the direct product subgroup $\mathrm{U}(3) \otimes$ $\mathrm{U}(\mathrm{n})$ of the $\mathrm{U}(3 n)$ symmetry group: $\mathrm{U}(3 n) \supset \mathrm{U}(3) \otimes \mathrm{U}(\mathrm{n})$. As it is well known U(3) is the set of all the unitary transformations in the three dimensional complex space of the coordinates and momenta. Similarly, U(n) is the group of all the unitary transformations in the $n$ dimensional pseudospace of the particle indices.

The physically relevant subgroups are those which contain (i) in the space part the angular momentum group $\mathrm{U}(3) \supset$ $\mathrm{SU}(3) \supset \mathrm{SO}(3)$ and (ii) the permutation group in the particle index space. The subgroups in the latter embedding are

$$
\mathrm{U}(n) \supset \mathrm{U}(n-1) \supset \mathrm{O}(n-1) \supset \mathrm{S}(n),
$$

where the decrease in dimension from $n$ to $n-1$ comes from the elimination of the center of mass motion [27].

The representations of the $U(3)$ space and $U(n)$ particle index groups are uniquely related to each other $[27,28]$ : if the irrep of $\mathrm{U}(3)$ has the labels $\left[h_{1}, h_{2}, h_{3}\right]$, then the corresponding irrep of $\mathrm{U}(\mathrm{n})$ is $\left[h_{1}, h_{2}, h_{3}, 0, \ldots, 0\right]$.

The treatment of the spin-isospin degrees of freedom in the particle formalism is very similar to that of the space degrees of freedom. Let $\chi_{\mu}^{s}$ denote the spin-isospin wave function of nucleon $s$, where $\mu=1,2,3,4$ stands for the four spinisospin states. In [27] the $\rho_{\mu}^{s}$ creation operators are introduced by the $\chi_{\mu}^{s}=\rho_{\mu}^{s}|0\rangle$ equation. The corresponding annihilation operators are $\theta_{\mu}^{s}$. Then the operators $\tilde{C}_{\mu \nu}^{s t}=\rho_{\mu}^{s}, \theta_{\nu}^{t}$ generate the $\mathrm{U}^{S T}(4 \mathrm{n})$ symmetry group of the $n$ nucleon system. The generators of the $\mathrm{U}^{S T}(4)$ and $\mathrm{U}^{S T}(n)$ subgroups $\left[\mathrm{U}^{S T}(4 \mathrm{n})\right.$ $\left.\supset \mathrm{U}^{S T}(4) \otimes \mathrm{U}^{S T}(n)\right]$ are obtained by contraction:

$$
\tilde{C}_{\mu \nu}=\sum_{s=1}^{n} \tilde{C}_{\mu \nu}^{s s}, \quad \tilde{C}^{s t}=\sum_{\mu=1}^{4} \tilde{C}_{\mu \mu}^{s t} .
$$

If the representation label of $\mathrm{U}^{S T}(4)$ is $\left[f_{1} f_{2} f_{3} f_{4}\right]$ then that of $\mathrm{U}^{S T}(n)$ has to be $\left[f_{1} f_{2} f_{3} f_{4}, 0, \ldots, 0\right]$

The physically relevant subgroup chains contain the $\mathrm{SU}^{S}(2)$ spin and $\mathrm{SU}^{T}(2)$ isospin groups and the $\mathrm{S}^{S T}(n)$ permutation group. The total antisymmetry of the n-nucleon system requires that the irreps of the $S(n)$ permutation group in the real three dimensional space and in the spin-isospin $S^{S T}(n)$ are uniquely related to each other: they are supposed to have associate representations (with Young patterns being mirror images of each other).

\section{B. Transformations between different configurations}

The transformation between different configurations amounts to redistribution of the nucleons; therefore, it can be studied within the particle scheme. In [2] it was presented in detail for the (simple) MUSY of the two binary cluster configurations. In that case it was shown that (i) MUSY is composed of the usual dynamical symmetries of each configuration, and a further symmetry which connects them, and (ii) the connecting symmetry was the Talmi-Moshinsky transformation between the two sets of Jacobi coordinates of an underlying three-cluster configuration.

Here one sees that the general MUSY, which connects any kind of cluster or shell configuration, has a similar structure. In particular, (i) each configuration has a U(3) dynamical symmetry, and (ii) the connecting symmetry is the invariance with respect to the transformations in the particle index space, which incorporates all the $(n)$ nucleons. This invariance is guaranteed when the physical operators are expressed in terms of the contracted U(3) operators of (1) and (18). In such a case the physical operators are totally symmetric with respect to the transformations in the particle index space, i.e., with respect to the transformation from one configuration to the other.

Please note that a more general Hamiltonian than that of Eq. (16) can also be invariant with respect to the transformations from one configuration to the other. In particular, the Hamiltonians expressed in terms of the operators of the group chain indicated in Eq. (16) are configuration independent, due to the reasons explained beforehand. The analytical solvability, like in the case of Eq. (16), corresponds to a special case.

\section{APPLICATION}

Here I show an illustrative application of the MUSY to the ${ }^{44} \mathrm{Ti}$. This nucleus has some well-established low-lying bands [29], and in addition a reliable experimental evidence is available for core-plus-alpha states forming four bands [30]. Furthermore, high-lying resonances are populated in the ${ }^{28} \mathrm{Si}+{ }^{16} \mathrm{O}$ reactions [31].

I describe the ground-state region by the shell model, more specifically by its simplified version of the quartet model (having only the $\mathrm{U}^{S T}$ (4) Wigner-scalar sector) [9]. The core-plus-alpha states and the ${ }^{16} \mathrm{O}$ resonances are treated as ${ }^{40} \mathrm{Ca}+{ }^{4} \mathrm{He}$ and ${ }^{28} \mathrm{Si}+{ }^{16} \mathrm{O}$ cluster states, respectively [21]. These configurations are supposed to have the building clusters in their ground intrinsic states.

The core-plus-alpha spectrum was already studied within the semimicroscopic algebraic cluster model in [32]. Here 


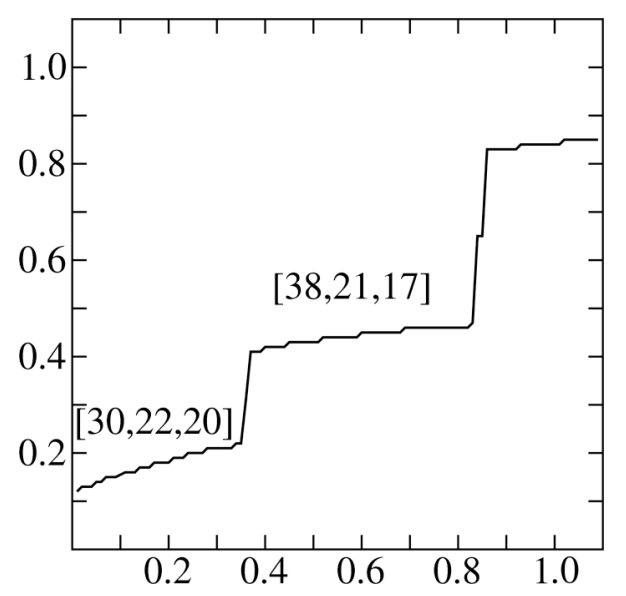

FIG. 1. Stability of the (quasidynamical) U(3) symmetry from Nilsson-model calculation [4]. The scale on the axes is given in $\beta$ deformation parameters: horizontal, input; vertical, output.

I show that it can be described together with the low-lying quartet (shell) spectrum and the highly excited ${ }^{28} \mathrm{Si}+{ }^{16} \mathrm{O}$ cluster states.

The low-lying bands [29] are associated to those of the leading SU(3) representations of the quartet model, which correspond to the largest deformation (and to the smallest energies). These are the $(12,0) 0^{+}$and $(8,2) 0^{+}$positive and $(13,3) 3^{-}$and $(11,4) 4^{-}$negative parity bands. (As for the negative parity bands, in the model space there is also a $(14,1) 1^{-}$band, but it has not yet been established experimentally).

The alpha-cluster bands correspond to the lowest-lying Pauli-allowed representations: $(12,0),(13,0),(14,0)$, and $(15,0)$, respectively.

Concerning the ${ }^{28} \mathrm{Si}+{ }^{16} \mathrm{O}$ cluster states, I associate their positive and negative parity bands with the leading representations of the $4 \hbar \omega$ and $5 \hbar \omega$ excitations. Around this excitation a shape isomer of the ${ }^{44} \mathrm{Ti}$ nucleus is expected according to the calculations [4] of the self-consistency and stability of the (quasidynamical) SU(3) symmetry (which is uniquely related to the quadrupole deformation). This method reveals the shape isomers as stability regions (horizontal plateaus) of the symmetry and/or quadrupole deformation. As shown in Fig. 1, there is some small fluctuation of the effective U(3) quantum numbers, as usual [4], but the stability region is well pronounced; actually it is even better than around the ground state.

As discussed beforehand, both the shell (quartet) and the (different) cluster configuration(s) have bases characterized by the representation labels of group chain (15). The difference of the configurations manifests itself in the sets of quantum numbers. MUSY is realized when the Hamiltonian is expressed only in terms of the contracted U(3) operators.

A particular form of the Hamiltonian that proved to be useful [7] is

$$
\hat{H}=\epsilon \hat{n}+a \hat{C}_{\mathrm{SU}(3)}^{(2)}+b \hat{C}_{\mathrm{SU}(3)}^{(3)}+d \frac{1}{2 \theta} \hat{L}^{2}
$$

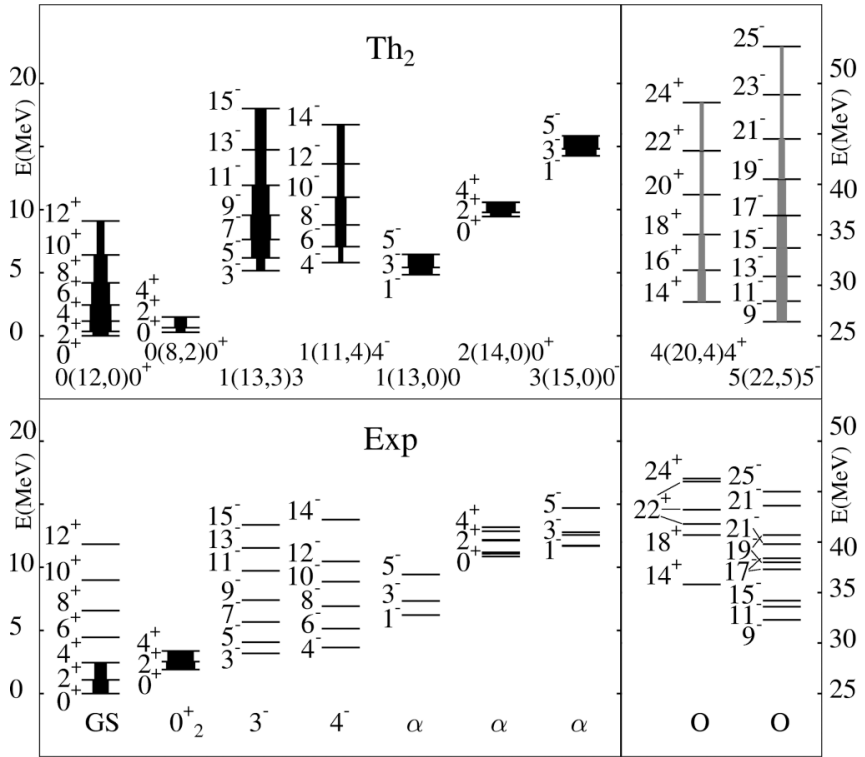

FIG. 2. Experimental spectrum of the ${ }^{44} \mathrm{Ti}$ nucleus in comparison with that of the multiconfigurational dynamical symmetry. The Hamiltonian of Eq. (24) is applied with two parameters. In the experimental spectrum GS stands for ground-state band, the wellestablished bands are denoted by their $\mathrm{K}^{\pi}$ labels, $\alpha$ indicates the alpha-cluster state, while $\mathrm{O}$ means ${ }^{28} \mathrm{Si}+{ }^{16} \mathrm{O}$ resonances. The model bands are characterized by the $n(\lambda \mu) \mathrm{K}^{\pi}$ quantum numbers, where $n$ shows the major shell excitations with respect to the ground state. The width of the arrow between the states is proportional to the strength of the $E 2$ transition. The real strength of the gray arrows $\left({ }^{16} \mathrm{O}\right.$ bands) is five times the strength of the illustrated ones.

Here $\theta$ is the moment of inertia calculated classically for the rigid shape determined by the $\mathrm{U}(3)$ quantum numbers (for a rotor with axial symmetry) [33]. The parameters $\epsilon, a, b$, and $d$ can be fitted to the experimental data.

I have performed calculations with a simplified form of Eq. (24), when $b=0$ and $d=1$ were fixed, i.e., there were only two parameters to fit (upper part of Fig. 2). It is remarkable that this extremely simple Hamiltonian (with $\epsilon=$ $4.845 \mathrm{MeV}$ and $a=-0.00425 \mathrm{MeV}$ ) reproduces the gross features of the energy spectra of three different configurations: quartet, core-plus-alpha, and ${ }^{28} \mathrm{Si}+{ }^{16} \mathrm{O}$ in a large range of energy and deformation. Including a third order term and fitting the rotational parameter gives a somewhat better approximation, of course. In the fitting procedure the weights of different states were taken as follows. The well-known states of the low-lying bands had 1.0; the states with uncertain spin parity (in parentheses in compilation [29]) had 0.5. When there is more than one candidate for the state in an alphacluster band, the unit weight was divided by their number, and the ${ }^{28} \mathrm{Si}+{ }^{16} \mathrm{O}$ resonances were taken with weight 0.5 .

The in-band $B(E 2)$ value is given as

$$
\begin{aligned}
B\left(E 2, L_{i} \rightarrow L_{f}\right)= & \frac{2 L_{f}+1}{2 L_{i}+1} \alpha^{2} \\
& \times\left|\left\langle(\lambda \mu) K L_{i},(11) 2||(\lambda \mu) K L_{f}\right\rangle\right|^{2} C_{\mathrm{SU}(3)}^{2},
\end{aligned}
$$


where $\left\langle(\lambda \mu) K L_{i},(11)\left(2 \|(\lambda \mu) K L_{f}\right\rangle\right.$ is a $\mathrm{SU}(3) \supset \mathrm{SO}(3)$ Wigner coefficient [34], and $\alpha^{2}(=0.361 \mathrm{~W} . \mathrm{u})$. is a parameter fitted to the experimental value of the $2_{1}^{+} \rightarrow 0_{1}^{+}$transition of 13 W.u.

\section{SUMMARY AND CONCLUSIONS}

In this paper I have presented the mathematical background of the multiconfigurational dynamical symmetry. This symmetry turns out to be the common intersection of the shell, collective, and cluster models for the multi-major-shell problem. Originally it was introduced on the basis of wavefunction and eigenvalue connections between the different configurations.

The MUSY results from the composition of two different symmetries. It contains a usual [U(3)] dynamical symmetry in each configuration, and another one, which connects the different configurations to each other.

From the viewpoint of its logical structure the MUSY is similar to some other composite symmetries of nuclear structure, including Wigner's supermultiplet theory [11] from the early days, as well as the more recent dynamical supersymmetry (SUSY) [35]. In the SUSY case, e.g., there is a bosonic and a fermionic sector, equipped with usual dynamical symmetries, and the supertransformations connect them to each other.

For the multiconfigurational symmetry the connecting transformations are those of the particle index pseudospace, which take one configuration to the other. So far it was known only for the simple case of the two binary cluster configurations. Here I have presented these transformations in the general form, connecting any configurations. Mathematically it requires the application of operators which are contracted with respect to the particle index. In order to make the discussion complete, I have incorporated also the wave-function and eigenvalue arguments in their general form.

Similarly to some other dynamical symmetries, the MUSY incorporates dual breaking of symmetries: its U(3) and SU(3) symmetries are dynamically broken, while its $\mathrm{SO}(3)$ symmetry is spontaneously broken in the intrinsic system (resulting in deformed shapes).
I have presented here an application of the MUSY to the ${ }^{44} \mathrm{Ti}$ nucleus. Its low-lying bands were described within a quartet (shell) model, together with the high-lying ${ }^{40} \mathrm{Ca}+{ }^{4} \mathrm{He}$ and ${ }^{28} \mathrm{Si}+{ }^{16} \mathrm{O}$ cluster spectra. The gross features of the spectrum distributed in different energy windows and deformation regions could be reproduced to a reasonable approximation with a very simple Hamiltonian. The ${ }^{28} \mathrm{Si}+{ }^{16} \mathrm{O}$ spectrum corresponds to a shape isomer around $4 \hbar \omega$ excitation, similarly to the ${ }^{12} \mathrm{C}+{ }^{16} \mathrm{O}$ states in ${ }^{28} \mathrm{Si}$.

As for the realization of the multiconfigurational symmetry in nature the present knowledge is very limited. Nevertheless, some of the first applications are promising. Due to the dynamical symmetry approximation it produces only simple spectra, of course. The description of the experimental details requires a fine tuning by taking into account symmetrybreaking terms both in the shell and collective, as well as in the cluster picture. Therefore, MUSY cannot compete with the detailed calculations of these structure models. Nevertheless, it could be useful even for the fully microscopic descriptions. In the large scale $a b$ initio calculations, e.g., the truncation scheme plays an essential role [36]. In this respect MUSY could also give some helpful insight in determining the physically most relevant set of bases.

On the other hand, studying the gross features of the spectra, and the interrelations of fundamental structure models, MUSY seems to be a very suitable tool. The unified description of the low-lying quartet spectrum and the high-lying cluster spectra of different configurations, e.g., seems to be a remarkable new feature of the structure studies. The possible clusterizations of the shape isomers, and their relation to the reaction channels, are also useful pieces of information.

Further investigations are highly desirable in order to reveal to what extent and in which area this unifying symmetry is valid.

\section{ACKNOWLEDGMENTS}

This work was supported by the National Research, Development, and Innovation Fund of Hungary, financed under the K18 funding scheme Project No. K 128729.
[1] J. Cseh, Phys. Rev. C 50, 2240 (1994).

[2] J. Cseh and K. Kato, Phys. Rev. C 87, 067301 (2013).

[3] J. Cseh, J. Phys. Conf. Ser. 580, 012046 (2015).

[4] J. Cseh, G. Riczu, and J. Darai, Phys. Lett. B 795, 160 (2019).

[5] J. Darai, J. Cseh, and D. G. Jenkins, Phys. Rev. C 86, 064309 (2012).

[6] J. Cseh, J. Darai, W. Sciani, Y. Otani, A. Lepine-Szily, E. A. Benjamim, L. C. Chamon, and R. L. Filho, Phys. Rev. C 80, 034320 (2009).

[7] J. Cseh and G. Riczu, Phys. Lett. B 757, 312 (2016).

[8] P. Adsley et al., Phys. Rev. C 95, 024319 (2017).

[9] J. Cseh, Phys. Lett. B 743, 213 (2015).

[10] J. P. Elliott, Proc. R. Soc. A 245, 128 (1958); 245, 562 (1958).

[11] E. P. Wigner, Phys. Rev. 51, 106 (1937).

[12] J. P. Elliott and P. G. Dawber Symmetry in Physics (MacMillan, London, 1986).
[13] J. P. Draayer, Y. Leschber, S. C. Park, and R. Lopez, Comput. Phys. Commun. 56, 279 (1989).

[14] J. P. Elliott and T. H. R. Skyrme, Proc. R. Soc. A 232, 561 (1955); K. T. Hecht, Nucl. Phys. A 170, 34 (1971).

[15] D. J. Rowe, Rep. Prog. Phys. 48, 1419 (1985).

[16] J. P. Draayer, in Algebraic Approaches to Nuclear Structure, edited by R. F. Casten Harwood (Academic, New York, 1993), p. 423.

[17] G. Rosensteel and D. J. Rowe, Phys. Rev. Lett. 38, 10 (1977); Ann. Phys. (NY) 126, 343 (1980).

[18] D. J. Rowe and G. Rosensteel, Phys. Rev. C 25, 3236(R) (1982).

[19] O. Castanos and J. P. Draayer, Nucl. Phys. A 491, 349 (1989).

[20] F. Iachello and A. Arima, The Interacting Boson Model (Cambridge University, Cambridge, England, 1987).

[21] J. Cseh, Phys. Lett. B 281, 173 (1992); J. Cseh and G. Lévai, Ann. Phys. (NY) 230, 165 (1994). 
[22] F. Iachello, Phys. Rev. C 23, 2778 (1981); F. Iachello and R. D. Levine, J. Chem. Phys. 77, 3046 (1982).

[23] K. Wildermuth and Th. Kanellopoulos, Nucl. Phys. 7, 150 (1958).

[24] J. Cseh, G. Levai, A. Algora, P. O. Hess, A. Intasorn, and K. Kato, Acta Phys. Hungarica New Series: Heavy Ion Phys. 12, 119 (2000).

[25] J. Cseh, Eur. Phys. J. A Spec. Top. 229, 2543 (2020).

[26] J. Cseh, Phys. Lett. B 793, 59 (2019).

[27] P. Kramer and M. Moshinsky, in Group Theory and its Applications, edited by E. M. Loebl (Academic, New York, 1968), p. 339.

[28] M. Moshinsky, J. Math. Phys. 4, 1128 (1963).

[29] https://www.nndc.bnl.gov/ensdf/EnsdfDispatcherServlet.

[30] S. Ohkubo et al., Prog. Theor. Phys. Suppl. 132, 1 (1998) and references therein; T. Yamaya, S. Oh-ami, M. Fujiwara, T. Itahashi, K. Katori, M. Tosaki, S. Kato, S. Hatori, and S.
Ohkubo, Phys. Rev. C 42, 1935 (1990); P. Guazzoni et al., Nucl. Phys. A 564, 425 (1993).

[31] U. Abbondanno, Trieste Report No. INFN/BE-91/11, 1991.

[32] J. Cseh, G. Levai, A. Intasorn, and K. Kato, Acta Univ. Debrecen. Ser. Phys. Chim. 38-39, 81 (2005).

[33] J. Cseh, G. Riczu, J. Darai, T. Dytrych, and E. Betak, Bulg. J. Phys. 44, 466 (2017).

[34] Y. Akiyama and J. P. Draayer, Comput. Phys. Commun. 5, 405 (1973).

[35] A. B. Balantekin, I. Bars, and F. Iachello, Phys. Rev. Lett. 47, 19 (1981); F. Iachello and P. Van Isacker, The Interacting Boson Fermion Model (Cambridge University, Cambridge, England, 1991); G. Lévai, J. Cseh, and P. Van Isacker, Eur. Phys. J. A 12, 305 (2001).

[36] T. Dytrych, K. D. Sviratcheva, J. P. Draayer, C. Bahri, and J. P. Vary, J. Phys. G 35, 123101 (2008); K. D. Launey, T. Dytrych, G. H. Sargsyan, R. B. Baker, and J. P. Draayer, Eur. Phys. J. Spec. Top. 229, 2429 (2020). 